\title{
Plurilinguisme : pluralité des problèmes, pluralité des approches
}

\section{Michèle Verdelhan-Bourgade}

\section{(2) OpenEdition}

\section{Journals}

Édition électronique

URL : http://journals.openedition.org/trema/246

DOI : 10.4000/trema.246

ISSN : 2107-0997

\section{Éditeur}

Faculté d'Éducation de l'université de Montpellier

\section{Édition imprimée}

Date de publication : 1 septembre 2007

Pagination : 5 - 16

ISSN : 1167-315X

\section{Référence électronique}

Michèle Verdelhan-Bourgade, «Plurilinguisme : pluralité des problèmes, pluralité des approches »,

Tréma [En ligne], 28 | 2007, mis en ligne le 30 septembre 2010, consulté le 08 janvier 2020. URL:

http://journals.openedition.org/trema/246; DOI : 10.4000/trema.246

Ce document a été généré automatiquement le 8 janvier 2020.

Trema 


\title{
Plurilinguisme : pluralité des problèmes, pluralité des approches
}

\author{
Michèle Verdelhan-Bourgade
}

1 En me souvenant de mes origines méridionales et en l'honneur de la langue d'oc, je pourrais dire abruptement: «Plurilinguisme, qu'es aco?» Horreur universitaire : comment peut - on, même en plaisantant et même en occitan, se demander ce qu'est le plurilinguisme alors qu'il est déjà fortement installé dans le lexique linguistique? Et pourtant cette interrogation peut avoir sa légitimité. D'une part, en effet, le public du colloque IUFM d'avril 2006 était très varié, et celui des lecteurs de TREMA l'est aussi : certains sont certes linguistes professionnels, d'autres pas du tout; dans ce contexte, un rappel sur l'origine et le contenu terminologique n'est pas forcément inutile. D'autre part, le mot traduit une notion qui s'accompagne d'incertitudes et flottements sur beaucoup de points. Comme pour tout terme à la mode, il convient donc de l'étudier avec précaution, quel que soit le

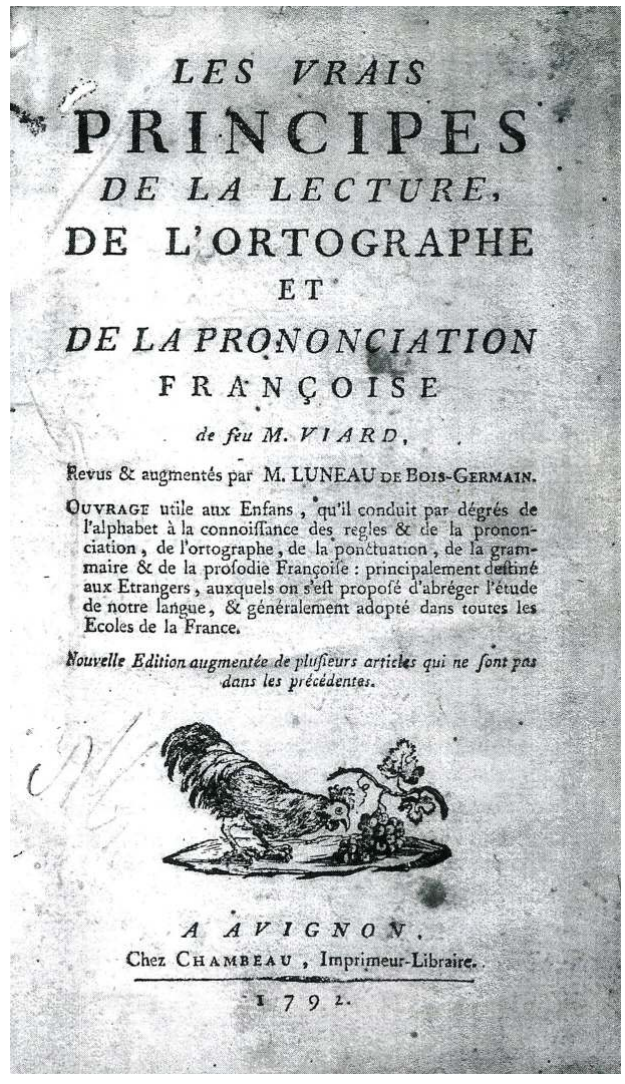
poids des institutions prestigieuses qui en font leur cheval de bataille.

2 Il s'agira donc ici de rappeler quelques fondamentaux du travail sur le plurilinguisme, d'en souligner les points forts aussi bien que les points litigieux, en même temps que poser des jalons pour une réflexion sur les enjeux liés à ce concept. 


\section{Incertitudes épistémologiques}

Le terme de plurilinguisme parait lumineux : pluri -, «plusieurs », ling - «langue». Et pourtant l'incertitude commence au cœur même du mot, dans la notion de langue, et se poursuit avec celle de pluralité.

\section{I.1. Le nombre de langues}

Combien y a - $\mathrm{t}$ - il de langues? Dans le monde, on en compterait 3000 selon M. MALHERBE (1983), 6000 selon le Dictionnaire de didactique, (CUQ, dir. 2003).

En Europe, il y a 20 langues officielles pour la Commission européenne, 30 pour M. MALHERBE (mais celui - ci fait aller l'Europe jusqu'au Caucase).

En France, le rapport CERQUIGLINI trouvait 75 langues pour l'ensemble du pays (y compris les DOM - TOM et les langues de l'immigration). On reviendra plus loin sur la situation française et sa complexité. Mais la nation française ne connaît officiellement qu'une langue.

Comment ces divergences sont - elles possibles?

Ces incertitudes sont sans doute liées à la définition de la «langue » (par rapport à variété, dialecte ...) et à la détermination de son statut (officiel, national, régional ...). Toutefois, comme le remarque Léon FLEURIOT, linguiste, à propos du gallo, langue régionale de Bretagne, peu importe qu'on l'appelle langue ou patois, l'essentiel est qu'on l'étudie! Solide bon sens! Simplement, est - ce la même chose de prôner un plurilinguisme français - anglais, ou français - russe, et un plurilinguisme français - gallo ou ndjuka - wayana (langues de Guyane)? Au plan strictement linguistique, sans doute. $\mathrm{Au}$ plan de la politique éducative, de la politique linguistique, et même au plan psycholinguistique, c'est beaucoup moins net.

\section{I.2. La terminologie}

Le terme " plurilinguisme » est à analyser dans un paradigme déjà riche, voire encombré.

Commençons par Bilinguisme, dont la définition a évolué au fil du temps, y compris dans les ouvrages de vulgarisation linguistique souvent utilisés par les enseignants ou les formateurs. Regardons par exemple les deux versions du Dictionnaire de didactique, déjà cité. En 2003, le bilinguisme c'est la « coexistence au sein d'une même personne ou d'une société de deux variétés linguistiques » (CUQ, dir. 2003, p. 36).

Mais en 1976, (GALISSON et COSTE dir. p. 69), c'était une « situation qui caractérise les communautés linguistiques et les individus installés dans des régions, des pays où deux langues (bilinguisme) et plus (multilinguisme $=$ plurilinguisme) sont utilisées concurremment ».

11 De 1976 à 2003, on est passé d'un bilinguisme de langues à un bilinguisme étendu aux variétés, ce qui assouplit considérablement la notion. On est passé également d'une définition géographique (région, pays) à une définition psycho - sociale (personne, 
société). On peut y voir le reflet de l'évolution de la linguistique en trente ans. Mais, au bout du compte, ne peut - on se demander si le bilinguisme est encore l'affaire de la linguistique? au contraire asymétrique. C'est ainsi que le bilinguisme prôné dans certains établissements français à l'étranger est asymétrique, dans la mesure où les élèves non francophones sont dans l'obligation de pratiquer le français langue d'enseignement, tandis que les francophones ne sont pas tenus de devenir bilingues dans la langue du pays.

14 - Selon la manière d'apprendre les langues par le locuteur, les situations d'apprentissage (LUDI, PY), on distinguera :

- le bilinguisme composé : l'apprentissage de la langue 2 est lié à un changement de contexte (immigration par ex.), et correspond donc à une successivité dans les expériences. Chaque langue représente ensuite une expérience de vie particulière.

- le bilinguisme coordonné, concernant les personnes vivant en contexte bi ou multilingue (ex. DOM - TOM). Plusieurs langues sont disponibles pour parler du même monde.

- Selon le résultat d'apprentissage (LAMBERT 1974), on parle alors de bilinguisme additif ou soustractif, voire de semilinguisme lorsque les performances de l'apprenant sont médiocres dans les deux langues, dont il ne maitrise aucune vraiment.

\section{Multilinguisme}

Le sens du terme multilinguisme était identique à celui de plurilinguisme en 1976, mais a été différencié depuis. Il désigne aujourd'hui la présence de plusieurs langues sur un même territoire. orme de multilinguisme sociétal standardisé qui compte au moins trois variétés linguistiques et dont la distribution complémentaire est basée sur une répartition fonctionnelle », par exemple Luxembourg, Singapour. La répartition entre les langues serait souvent liée à une échelle de prestige.

\section{Plurilinguisme}

19 Le plurilinguisme serait - il du bilinguisme étendu à plusieurs langues? Ce n'est pas si simple.

20 Toujours selon la même référence, (CUQ, dir. 2003, p. 195), le plurilinguisme est la " capacité d'un individu d'employer à bon escient plusieurs variétés linguistiques », ce qui conforte la dimension psychologique déjà entrevue dans la définition du bilinguisme. On note ensuite que l'utilisation de la langue est liée à un modèle social et de nombreux facteurs situationnels (statut des participants, enjeux, types de discours, etc.), d'où la 
nécessité de mettre en jeu « une forme spécifique de compétence de communication ». La principale manifestation chez l'individu consiste en l'alternance codique.

Il apparait que le plurilinguisme est un concept plus souple que les précédents, qui comporte une dimension linguistique (contacts de langues), sociolinguistique (rôle des contextes de l'alternance codique), psycholinguistique (raisons des choix des locuteurs, rôle des émotions, de l'affectivité ...). C'est ce concept qui fonde actuellement la politique linguistique de l'Europe.

En quelque sorte, dans une Europe multilingue de fait, la politique européenne vise au plurilinguisme des habitants, c'est - à - dire qu'elle souhaite des individus passant sans heurt d'une langue à l'autre, (avec au moins deux langues, donc des bilingues) selon les contextes, les situations, voire les humeurs! (cf. définition du plurilinguisme dans le Cadre Européen Commun de référence, p. 12).

Le modèle avoué est un individu doué de compétence plurilingue, s'appuyant sur plusieurs langues pour communiquer et s'exprimer. Cette vision idéale sous - tend les mesures adoptées par le Conseil de l'Europe, notamment le Cadre Européen Commun de Référence, et le portfolio, sur lesquelles nous reviendrons.

\section{L'éventail embrouillé des situations linguistiques françaises}

Comment caractériser de ce fait la situation française?

Des statuts différents qui entraînent des situations d'apprentissage différentes.

L'IUFM, ayant pour mission de former des enseignants pour la France, un recentrage sur le pays permettra d'examiner les situations linguistiques des élèves, auxquelles ces enseignants peuvent se trouver confrontés. Et d'ailleurs la France en fournit déjà un très large éventail, dont on ne souhaite pas ici bien sûr faire un inventaire exhaustif. Ce bref survol permet de comprendre la variété des contacts de langues sur le territoire français.

\section{II.1. En France métropolitaine, un riche éventail de situations}

On les énumèrera rapidement.

- Cas des enfants de couples binationaux et / ou bilingues : les enfants parlent souvent les deux langues, mais aussi peuvent en avoir une comme langue d'usage, tout en comprenant assez bien et parlant plus ou moins la seconde. Ils peuvent être scolarisés dans une langue, parfois deux (en établissement bilingue quand ils existent).

- Sont aussi scolarisés en établissement bilingue des enfants monolingues d'origine, dont les parents considèrent comme une richesse de leur faire parler deux langues très tôt.

- Cas des enfants dans les milieux de langues régionales (catalan, occitan, basque, breton...). Cette langue régionale peut être :

- la langue de la communication familiale et de l'école (calandretas, bressoles, eskoli, diwan... ).

- la langue de la seule communication sociale, l'école se faisant en français. 
- La langue régionale est souvent l'objet d'une valorisation dans le milieu social et la région, les communications sociales se faisant dans cette langue.

- Cas des enfants de milieux issus de l'immigration récente. La langue 1 peut être la langue de la communication familiale (le plus souvent) mais pas de l'école, qui se fait en français. La langue d'origine n'est pas forcément objet de valorisation en dehors du cercle social restreint, bien que cela soit à nuancer selon les langues et les milieux sociaux. Et les parlers mixtes issus du mélange des langues (origine + français) sont plutôt stigmatisés au - dehors par les francophones.

- Cas des enfants scolarisés en français, qui apprennent une langue vivante étrangère (LVE) dès l'école, en raison des programmes officiels mis en place depuis quelques années. La terminologie institutionnelle a fluctué, entre sensibilisation, initiation, apprentissage ...

\section{II.2. Dans les DOM - TOM, la situation est elle - même complexe}

Les départements DOM présentent des situations très différentes.

- En Martinique et Guadeloupe, deux langues principales sont en présence, le créole et le français.

- En Guyane, on compte près de 30 langues (on ne sait pas trop), dont une dizaine de langues de l'immigration. Le français est langue seconde à deux niveaux :

- pour les habitants guyanais non francophones (créoles, amérindiens, noirs marrons..).

- pour les immigrés (du Surinam ou du Guyana, ou du Brésil), qui ne parlent aucune des langues de Guyane.

Les territoires TOM, dont le français est la langue officielle et langue de scolarisation, sont également très différents :

- Wallis et Futuna, deux îles perdues dans le Pacifique. En Polynésie, on a souvent la répartition une île - une langue. C'est le cas ici, il n'y a pas d'immigration.

- Mayotte possède deux langues locales, le kibushi (d'origine malgache) et le shimaoré, auquel on peut ajouter le comorien du fait de l'immigration.

- La Nouvelle Calédonie compte 28 langues mélanésiennes environ, ainsi que des langues de l'immigration (indonésien, vietnamien).

La France est donc bien un pays multilingue (G. VERMÈS) de fait, alors qu'il se présente comme monolingue de droit. Mais surtout on remarque que les contacts de langues recouvrent des statuts différents qui peuvent entraîner des situations d'apprentissage différentes. Quels enjeux éducatifs sont présents dans ce plurilinguisme?

\section{Des enjeux éducatifs différents}

Les facteurs de différenciation des situations énumérées ci - dessus sont de plusieurs ordres :

- nombre et statut des langues en contact (de 2 à 30 sur une même portion de territoire), degré de pratique ... 
- valorisation ou non des langues, qui peut entraîner des problèmes identitaires et culturels (lesquels ont aussi d'autres sources).

- scolarisation ou non dans la langue.

\section{III.1. Ces trois facteurs déterminent en gros les axes de recherche actuels sur le plurilinguisme}

- L'axe sociolinguistique des contacts de langue (descriptif des situations, des phénomènes linguistiques, de l'alternance codique ...). Le changement de code chez des individus maniant au moins deux langues a un caractère fascinant, et on ne peut pas forcément repérer quel critère sert pour l'usage de l'une ou l'autre langue et le passage du relais.

- L'axe psycholinguistique, intéressé par le sujet en action dans la langue et par le caractère identitaire de l'activité langagière.

- L'axe culturel, sur le thème de la rencontre et du dialogue des cultures. S'est développée depuis longtemps une réflexion sur le rôle du culturel dans l'apprentissage et la notion d'interculturel.

- L'axe méthodologique, sur la question de l'enseignement des LVE et de la langue de la scolarisation. On se demande alors quelle méthodologie convient pour telle situation d'apprentissage.

\section{III.2. En fonction de ces facteurs, il n'y a pas les mêmes enjeux d'apprentissage plurilingue} parler, lire, écrire, les compétences et connaissances scientifiques ou non linguistiques
(DNL) dans cette langue. Le tableau ci - dessous résume les différences de ces deux situations.

De la langue vivante étrangère à la langue de scolarisation : des besoins d'apprentissage décalés

\begin{tabular}{|l|l|l|}
\hline Domaine considéré & Elève en classe LVE & Elève nouvel arrivant en France \\
\hline \hline Oral / Ecrit & $\begin{array}{l}\text { Priorité à l'oral parlé, chanté } \\
\text { Contenu : société française }\end{array}$ & $\begin{array}{l}\text { Priorité à l'oral compris Contenu : la } \\
\text { situation scolaire Gros besoin d'écrit }\end{array}$ \\
\hline $\begin{array}{l}\text { Fonctionnement de la } \\
\text { communication }\end{array}$ & $\begin{array}{l}\text { Pas de situation nouvelle } \\
\text { Cadre scolaire d'origine }\end{array}$ & $\begin{array}{l}\text { École = milieu nouveau Règles } \\
\text { nouvelles de communication }\end{array}$ \\
\hline Contenus culturels & $\begin{array}{l}\text { Information externe Pas } \\
\text { d'enjeu identitaire }\end{array}$ & $\begin{array}{l}\text { Assimilation nécessaire d'une culture } \\
\text { scolaire (institution) et sociale. }\end{array}$ \\
\hline Système de la langue & $\begin{array}{l}\text { Apprentissage non prioritaire, } \\
\text { subordonné } \\
\text { communication à } \quad l a ~\end{array}$ & $\begin{array}{l}\text { Risque de rentitaire } \\
\text { Apprentissage prioritaire }\end{array}$ \\
\hline
\end{tabular}




\begin{tabular}{|l|l|l|}
\hline $\begin{array}{l}\text { Français et autres } \\
\text { disciplines }\end{array}$ & Sans objet. LVE pour LVE. & $\begin{array}{l}\text { Langue de scolarisation Pour le } \\
\text { français Pour l'école Pour les } \\
\text { concepts Pour des savoirs }\end{array}$ \\
\hline
\end{tabular}

Comme on le voit, ce ne sont pas les mêmes urgences, les mêmes besoins, ce ne sont pas mêmes contenus d'enseignement ni les mêmes progressions, et ce ne devrait pas être fait selon les mêmes méthodes.

\section{Des conséquences méthodologiques}

\section{IV.1. Trois visions (entre autres) en concurrence, concernant l'apprentissage des langues}

- La vision de l'apprentissage des langues comme celui de la multiplication de compétences monolingues. LVE 1, LVE2 et langue maternelle (LM) fonctionnent comme des tuyaux parallèles, mais distincts.

- La vision corollaire des dangers représentés dans l'apprentissage de la nouvelle LVE par la connaissance du système précédent (notamment LM) conduit à la peur des interférences. Les langues sont vues en opposition.

- La vision inverse conçoit une complémentarité des langues dans l'apprentissage, à travers la formation de l'interlangue, qui s'appuie beaucoup sur la langue maternelle. La notion d'interlangue a ainsi ouvert la voie à celle de compétence bilingue et plurilingue. C'est cette dernière vision qui domine actuellement, à travers l'individu idéalement bi - ou pluri lingue, bien que des études commencent à montrer les limites de cette approche.

\section{IV.2. Des tentatives pédagogiques différenciées selon les terrains et les situations}

On citera par exemple :

- Le programme EVLANG (1998 - 2000), dirigé par Michel CANDELIER, qui visait auprès des élèves la comparaison des langues et des cultures, la sensibilisation à la diversité, la formation d'une conscience linguistique ...

- Le portfolio européen, qui n'est pas une méthodologie, mais un état des lieux pour une personne. Le passeport pour les langues pour adultes de plus de 16 ans est un bilan linguistique selon l'échelle du Cadre Européen Commun de Référence (CECR) qui comporte une biographie langagière, les diplômes obtenus dans les langues, etc. Un passeport a été mis en place également pour les enfants.

- Le français langue de scolarisation (FLSCo), essai de constitution d'une didactique du français en milieu plurilingue, en vue de la scolarisation (DAVIN 2005, VERDELHAN).

Une constante se retrouve dans les travaux actuels: l'enseignement des langues prend appui sur langue d'origine, la L1. Un effort est fait en matière de valorisation, de comparaison, d'attitude positive à créer chez l'apprenant, afin d'éviter blocages et rejets identitaires. 


\section{IV.3. Le CECR, intérêt et limites}

\section{d} linguistiques, quel que soit le contexte. Mais il ne concerne en réalité que l'enseignement des LVE. Tous les exemples donnés au niveau A1, pour la compréhension de l'oral ou de l'écrit, relèvent du comportement en langue étrangère dans un pays étranger. La finalité scolaire est absente. De même, on ne voit pas de descripteurs prévus pour A1 et A2 en matière de compréhension de l'oral en tant qu'auditeur ; or c'est un problème majeur pour l'intégration scolaire des nouveaux arrivants.

On peut en outre se demander quel sera l'effet - retard d'une généralisation massive d'une même approche méthodologique et conceptuelle. Dans le chapitre 6, les auteurs se défendent d'imposer une démarche unique. Mais la forme des questions posées est trompeuse (à peine). Ex. p. 114 : « les utilisateurs du CECR envisageront et expliciteront selon le cas les moyens inventoriés ci - dessus (ou d'autres) qu'ils mettent en œuvre pour développer des compétences générales ... ». La formulation suppose que les utilisateurs raisonnent en termes de compétences générales telles que posées au début (notamment 
les savoir - être et savoir - apprendre, dont par ailleurs le Cadre nous dit qu'ils sont tautologiques).

\section{Enjeux idéologiques}

Le plurilinguisme prôné actuellement semble bien prendre le relais du Niveau - Seuil des années 1975, par besoin de mobilité des gens à travers l'Europe et d'adaptabilité aux emplois. Comme cela a été le cas dans le passé, cette étape nouvelle dans l'enseignement des langues est fortement liée aux besoins économiques et aux évolutions géopolitiques. Quel que soit son intérêt, elle comporte néanmoins un certain nombre de risques.

Danger 1: se contenter pour la plupart des apprenants d'une facilité de communication superficielle, mettant certes en œuvre des modes de communication pris dans des langues différentes, mais au détriment d'un apprentissage approfondi de chaque langue.

Danger 2 : la formation d'un plurilinguisme souple et diversifié s'accommode - $t$ - elle d'une méthodologie et d'une pensée conceptuelle unique?

Le plurilinguisme nouvelle cuvée est recherché au travers d'une globalisation conceptuelle massive. L'auteur de ces lignes est très circonspecte vis - à - vis des armes massives, même de construction! De plus, n'y a - $t$ - il pas une contradiction interne entre une vision du plurilinguisme comme souplesse et diversité et un Cadre commun qui vise à une certaine uniformisation?

Danger 3 : développer une idéologie du multiculturalisme au détriment d'une politique de creuset (HAMERS, 1997), voire au détriment des fondamentaux de l'éducation dans certains pays. Il est navrant d'assister, dans des pays où les besoins primordiaux d'éducation sont immenses et très loin d'être satisfaits, des gouvernants imposer le Cadre comme référence aux programmes du pays, alors qu'il est conçu pour des publics et des contextes radicalement différents.

Danger 4 : imposer une vision de l'apprenant qui ne serait pas conforme à une réalité, mais à des objectifs idéologiques. Le plurilinguisme est en effet vu comme un bienfait, et l'individu plurilingue est efficace, harmonieux, bien installé dans une Europe dans laquelle il trouve facilement $\mathrm{du}$ travail. Or des voix discordantes se font entendre, concernant l'utilité du bi - plurilinguisme (ESSER, 2006), pour l'individu dans son intégration scolaire ou sociale.

\section{Conclusion}

Un mouvement général se dessine vers un bi / plurilinguisme des individus, dans lequel apparaitraient trois niveaux (HAMERS) :

- une langue maternelle ou d'usage

- une langue de scolarisation

- une langue de communication internationale (ou plusieurs). 
La France aurait donc un double enjeu en matière de plurilinguisme :

- Réussir un plurilinguisme endogène, c'est - à - dire permettre à sa population qui a une langue 1 autre que le français de s'insérer par le plurilinguisme dans la société française. Ceci concerne les situations de langues régionales et locales, ainsi que les langues de l'immigration.

- Bâtir un plurilinguisme exogène, européen, voire mondial, par l'apprentissage à ses écoliers des langues vivantes étrangères.

Vaste programme. Le problème 1 est loin d'être réglé et obère fortement le fonctionnement de l'école, de manière différente selon les lieux considérés. Il pèse également sur la vie sociale et politique. Peut - on viser directement le plurilinguisme 2, celui de la communication européenne, souhaité par les tenants du cadre? Peut - être. Mais il serait sans doute dangereux de vouloir construire le second en négligeant le premier, qui est la base de la stabilité sociale.

\section{BIBLIOGRAPHIE}

BERTRAND, M. (1997). Les poupées russes des minorités nationales, ou quand la langue à apprendre est à la fois majoritaire et minoritaire, in Lefebvre et Hily, p. 127 - 142.

CANDELIER, M., (dir.) 2003. Evlang - l'éveil aux langues à l'école primaire. Bruxelles : De Boeck Duculot.

CASTELLOTTI, V. (2005). La langue maternelle en classe de langue étrangère. Paris, CLE International. CASTELLOTI, V. \& MOORE, D. (1997). (Dir.) Alternance des langues et apprentissages, éla, nº 108. Paris : Didier.

CAVALLI, M. (2005). Education bilingue et plurilinguisme. Paris : Hatier, coll. LAL.

CONSEIL de L'EUROPE. (2000). Un cadre européen commun de référence : apprendre, enseigner, évaluer. Strasbourg.

COSTE, D., MOORE, D., \& ZARATE, G. (1997). Compétence plurilingue et pluriculturelle. Strasbourg, Conseil de l'Europe.

COSTE, D. (1997). Éduquer pour une Europe des langues et des cultures, Etudes de linguistique appliquée 98. Paris, Didier.

CHRIST, I \& COSTE, D. (Dir.) (1995). Aspects de l'enseignement bilingue. éla, n 96. Paris : Didier.

CUQ, J. - P. (Dir.) (2003) Dictionnaire de didactique du français langue étrangère et seconde. Paris: ASDIFLE - CLE International.

CUMMINS, J., SWAIN, M. (1986). Bilingualism in Education. London, Longman. 
DAVIN, F. (2005). Didactique du français langue seconde en France. Le cas de la discipline " français » enseignée au collège. Thèse de Doctorat. Aix - Marseille.

ESSER, H. (2006). Sprache und Integration. Francfort : Campus - Verlag.

GAJO, L. (2000). « Le bilinguisme par l'apprentissage et le bilinguisme pour l'apprentissage ». Le français dans le monde, recherches et applications, Actualité de l'enseignement bilingue.Paris, CLE international.

GALISSON, R., \& COSTE, D. (Dir.) (1976). Dictionnaire de didactique des langues. Paris : Hachette.

HAMERS, J. F. (1997). Les situations plurilingues et leurs enjeux, in Lefebvre \& Hily, 1997, Les situations plurilingues et leurs enjeux, p. 3 - 22.

HAMERS, J. F., \& BLANC, M. (1983). Bilingualité et bilingualisme. Bruxelles : Mardaga.

KLEIN W., 1989, L'acquisition de langue étrangère, Paris Armand Colin. Traduit de l'allemand, 1987.

LAMBERT, W. E. (1974). Culture and language as factors in learning and education, F. E. Aboud \& R. D. Meade (Dir.) Culturals factors in Learning. Bellingham : Western Washington State College.

LEFEBVRE, M. - L. , \& HILY, M. - A. (dir.) (1997). Les situations plurilingues et leurs enjeux. Paris : L'Harmattan.

LUDI, G.,\& PY, B. (1986). Être bilingue. Berne, Peter Lang.

MALHERBE, M. (1983). Les langages de l'humanité. Paris : Seghers.

MOORE, D. (2005). Plurilinguisme et école. Paris, Hatier, LAL.

ROULET, E. (1980). Langue maternelle, langue seconde, vers une pédagogie intégrée. Paris, Hatier/ CREDIF, LAL.

SIGUAN, M., \& MACKEY, W. (1986). Education et plurilinguisme. Paris, Lausanne, Delachaux et Niestlé.

VERDELHAN - BOURGADE, M. (2002). Le français de scolarisation. Pour une didactique réaliste. Paris : PUF

VERMES, G., \& BOUTET, J. (1987). France, pays multilingue. Paris, L'Harmattan.

VERONIQUE, G.D. (2000). (Dir.) Didactiques de langues étrangères et recherches sur l'acquisition, éla, $\mathrm{n}^{\circ} 120$. Paris : Didier.

\section{RÉSUMÉS}

La mise en vedette actuelle de la notion de plurilinguisme, à tout bout de champ, ne doit pas occulter le fait qu'elle s'accompagne d'un certain flou dans plusieurs domaines. L'article présente brièvement la notion et les notions parentes. Il examine ensuite la situation française et sa richesse en matière de contacts de langues, qui conduit à une diversité d'enjeux langagiers, méthodologiques, éducatifs, voire idéologiques. L'incitation européenne à une méthodologie unique pour toute situation bi - ou plurilingue peut susciter des réserves au vu de la grande diversité des cas et des besoins.

Despite the ubiquitous use of the term "multilingualism," its meaning remains unclear. In this article, I will briefly present the concept and its intellectual genealogy. I will then introduce the French case, which is particularly rich in linguistic diversity, thereby allowing the exploration of a multiplicity of linguistic, methodological, educational, and ideological stakes. I will use this case 
study to argue that Europe's attempts to create a single methodology for all bi - or multi - lingual situations are ill - suited to the complexity of its social landscape and linguistic needs.

INDEX

Keywords : bilingualism, french for school purpose, methodology, plurilingualism, stake Mots-clés : bilinguisme, enjeu, français de scolarisation, méthodologie, plurilinguisme

\section{AUTEUR}

\section{MICHĖLE VERDELHAN-BOURGADE}

Professeur, Sciences du Langage, université Paul Valéry - Montpellier III 